\section{DYSLIPIDAEMIA \\ STATIN THERAPY IN MEN VERSUS WOMEN}

LDL-cholesterol lowering with statin therapy reduces cardiovascular events and all-cause mortality as effectively and safely in women as in men. This finding comes from a meta-analysis performed by the Cholesterol Treatment Trialists' (CTT) Collaboration and published in The Lancet. The efficacy of statins for lowering the LDL-cholesterol level and cardiovascular risk is well established. However, whether the effects of these drugs differ between the sexes was uncertain, mainly because women tend to be under-represented in statin trials. Therefore, the CTT collaborators performed a meta-analysis of 22 trials of statin therapy versus control and a further five trials of high-intensity versus low-intensity statin therapy. In total, these trials involved 174,149 participants, 46,675 (26.8\%) of whom were women, and the investigators had access to individual participant data. The median duration of follow-up in the trials was 4.9 years.

After adjustment for differences in baseline cardiovascular risk, each $1 \mathrm{mmol} / \mathrm{I}$ reduction in LDL cholesterol with statin therapy reduced the risk of vascular events similarly in women (rate ratio [RR] 0.84) and men (RR 0.78; adjusted heterogeneity $P=0.33$ ). No sexspecific differences were observed in the proportional effect of statins on the risk of major coronary events (RR 0.83 in women versus RR 0.74 in men; $P=0.10$ ), coronary revascularization (RR 0.76 in women versus RR 0.75 in men; $P=0.15$ ), or stroke (RR 0.90 in women versus RR 0.83 in men; $P=0.31$ ).

Statin therapy was associated with similar reductions in mortality for both men and women. For each $1 \mathrm{mmol} / \mathrm{l}$ lowering of LDL cholesterol, vascular death was reduced by $8 \%$ in women and $13 \%$ in men $(P=0.84)$, and all-cause death was reduced by $9 \%$ in women and $10 \%$ in men $(P=0.43)$. No sex-specific differences were observed in the incidence of cancer or in cancer mortality. The investigators note that the "benefits greatly outweigh the known hazards of statin therapy", such as myopathy, rhabdomyolysis, and incident diabetes mellitus. They conclude, therefore, that statins are "an effective means to prevent [cardiovascular] disease among women as well as men".

Gregory B. Lim

Original article Cholesterol Treatment Trialists' (CTT) Collaboration. Efficacy and safety of LDL-lowering therapy among men and women: meta-analysis of individual data from 174000 participants in 27 randomised trials. Lancet doi:10.1016/S0140-6736(14)61368-4 